\title{
EXISTENCE AND UNIQUENESS OF THE SOLUTION TO THE MODIFIED SCHRÖDINGER MAP
}

\author{
JUN KATO
}

\begin{abstract}
We prove the local existence of the solution to a certain system of nonlinear Schrödinger equations arising from Schrödinger maps for the initial data in $H^{s}\left(\mathbf{R}^{2}\right)$ with $s>1 / 2$. The uniqueness of the solution is also proved when the data belong to $H^{1}\left(\mathbf{R}^{2}\right)$.
\end{abstract}

\section{Introduction}

We consider the initial value problem for the system of the nonlinear Schrödinger equations in two space dimensions

$$
(\mathrm{MSM})\left\{\begin{array}{c}
i \partial_{t} u_{1}+\Delta u_{1}=-2 i A \cdot \nabla u_{1}+B u_{1}+|A|^{2} u_{1} \\
\quad \pm i \operatorname{Im}\left(u_{2} \bar{u}_{1}\right) u_{2}, \quad(t, x) \in(0, T) \times \mathbf{R}^{2} \\
i \partial_{t} u_{2}+\Delta u_{2}=-2 i A \cdot \nabla u_{2}+B u_{2}+|A|^{2} u_{2} \\
\pm i \operatorname{Im}\left(u_{1} \bar{u}_{2}\right) u_{1}, \quad(t, x) \in(0, T) \times \mathbf{R}^{2} \\
u_{1}(0, x)=u_{0}^{1}(x), \quad u_{2}(0, x)=u_{0}^{2}(x), \quad x \in \mathbf{R}^{2},
\end{array}\right.
$$

where $u_{1}, u_{2}$ are complex valued functions (we set $u=\left(u_{1}, u_{2}\right)$ in the following), and $A=\left(A_{1}[u], A_{2}[u]\right), B=B[u]$ are defined by

$$
\begin{aligned}
& A_{j}[u]= \pm 2 G_{j} * \operatorname{Im}\left(u_{1} \bar{u}_{2}\right), \quad j=1,2, \\
& G_{1}(x)=\frac{1}{2 \pi} \frac{x_{2}}{|x|^{2}}, \quad G_{2}(x)=-\frac{1}{2 \pi} \frac{x_{1}}{|x|^{2}}, \\
& B[u]=\mp \sum_{j, k=1}^{2} 2 R_{j} R_{k} \operatorname{Re}\left(u_{j} \bar{u}_{k}\right) \mp 2|u|^{2} .
\end{aligned}
$$

Here, for a complex number $z, \operatorname{Re} z$ and $\operatorname{Im} z$ denotes the real part of $z$ and the imaginary part respectively, and $R_{j}=\partial_{j}(-\Delta)^{-1 / 2}$ denotes the Riesz transforms. We notice that

$$
\operatorname{div} A=0 \quad \text { and } \quad \operatorname{rot} A=\partial_{1} A_{2}-\partial_{2} A_{1}= \pm 2 \operatorname{Im}\left(u_{1} \bar{u}_{2}\right)
$$

hold from the definition of $A$. These properties are used effectively to construct the solution to (MSM) for the low regularity initial data.

Received June 9, 2004.

JSPS Research Fellow. 
The system (MSM) above is called the modified Schrödinger map which is derived by Nahmod-Stefanov-Uhlenbeck [11] from Schrödinger maps from $\mathbf{R} \times \mathbf{R}^{2}$ to the unit sphere $S^{2}$ or to the hyperbolic space $\mathbf{H}^{2}$ by using appropriate gauge change. The sign \pm corresponds to the each case respectively. As for the modified Schrödinger map, Nahmod-Stefanov-Uhlenbeck [12] showed the existence and uniqueness of the solution for the data $u_{0} \in H^{s}\left(\mathbf{R}^{2}\right)$ with $s>1$ by using the energy method. In this paper, we show the improvement of their result.

Theorem 1.1. Let $u_{0} \in H^{s}\left(\mathbf{R}^{2}\right)$ with $s>1 / 2$. Then, there exists $T>0$ satisfying

$$
\min \left\{1, C /\left(\left(1+\left\|u_{0}\right\|_{L^{2}}^{q}\right)\left\|u_{0}\right\|_{H^{s}}^{q}\right)\right\} \leq T \leq 1
$$

and at least one solution $u \in L^{\infty}\left(0, T ; H^{s}\right) \cap C\left([0, T] ; L^{2}\right)$ to (MSM) such that

$$
J^{\delta} u \in L^{p}\left(0, T ; L^{q}\right),
$$

where $J^{\delta}=(I-\Delta)^{\delta / 2}, s-1 / 2>\delta>2 / q>0$, and $1 / p=1 / 2-1 / q$.

Remark 1.2. (1) The modified Schrödinger map is invariant with respect to the scale transformation

$$
u(t, x) \mapsto \lambda u\left(\lambda^{2} t, \lambda x\right), \quad \lambda>0 .
$$

Then, the scaling argument suggests that the critical space for the local wellposedness of the Cauchy problem (MSM) is $L^{2}\left(\mathbf{R}^{2}\right)$. We also notice that (MSM) conserves the $L^{2}$-norm (see Proposition 2.5 below).

(2) Since the modified Schrödinger map is derived as the first order derivatives of the original Schrödinger map (see [11, Theorem 2.1]), the local well posedness of (MSM) in $H^{s}$ corresponds to the local well-posedness of the original Schrödinger map in $H^{s+1}$.

(3) As is pointed out in $[11, \S 3]$, it is not possible to go back directly from the solution of the (MSM) to the original Schrödinger map. However, a priori estimate and the estimate on the time of existence on the smooth solution to (MSM) are made use of in order to construct the low regularity solution to the Schrödinger map. See $[11, \S 3]$ for details.

(4) After this work was completed, the author was informed that Kenig-Nahmod obtained the similar result for the Ishimori system ([7]). For the Ishimori system, see [9] and references therein. It is known that the Ishimori system is a generalization of the Heisenberg model for a ferromagnetic spin system, which is equivalent to the Schrödinger map in the case where the target manifold is $S^{2}$ (see $[4, \S 1])$.

In Theorem 1.1, the uniqueness of the solution is not obtained. However, for the uniqueness of the solution to (MSM) we obtain the following result by using the Vladimirov's argument [17] (see also [14]). 
Theorem 1.3. Let $u_{0} \in L^{2}\left(\mathbf{R}^{2}\right)$. We assume that $u$ and $v$ are solutions to $(M S M)$ on $(0, T) \times \mathbf{R}^{2}$ in the distribution sense with the same data $u_{0}$ satisfying

$$
\begin{gathered}
u, v \in C\left([0, T] ; L^{2}\left(\mathbf{R}^{2}\right)\right), \\
\|u\|_{L_{T}^{\infty} H_{x}^{1}} \leq M, \quad\|v\|_{L_{T}^{\infty} H_{x}^{1}} \leq M,
\end{gathered}
$$

for some constant $M>0$. Then, we have $u(t)=v(t)$ in $L^{2}\left(\mathbf{R}^{2}\right)$ for $0 \leq t \leq T$.

Corollary 1.4. If we assume $u_{0} \in H^{1}\left(\mathbf{R}^{2}\right)$, then the solution in the class of Theorem 1.1 is uniquely determined.

For the proof of Theorem 1.1 we use the compactness argument. Because the local well-posedness for smooth data is already known, our task is to show a priori estimate for the solution to (MSM). To recover the loss of the derivatives caused by the nonlinearity, the following type of estimate

$$
\left\|J^{s} w\right\|_{L_{T}^{p} L_{x}^{q}} \lesssim\|w\|_{L_{T}^{\infty} H_{x}^{s+1 / 2+\varepsilon^{\prime}}}+\|F\|_{L_{T}^{2} H_{x}^{s-1 / 2}}
$$

for the solution to $i \partial_{t} w+\Delta w=F$ is crucial in our argument, where $p, q$ are the admissible exponent for Strichartz estimates (see Proposition 2.6 below for the precise statement). Compared with the usual Strichartz estimate

$$
\left\|J^{s} w\right\|_{L_{T}^{p} L_{x}^{q}} \lesssim\|w(0)\|_{H^{s}}+\|F\|_{L_{T}^{1} H_{x}^{s}},
$$

estimate (1.5) says that we have a gain of the regularity $1 / 2$ on the inhomogeneous term at the cost of a loss of the regularity $1 / 2+\varepsilon^{\prime}$ on the homogeneous term. This type of estimate is first appeared in Koch-Tzvetkov [10] in the context of the Benjamin-Ono equation.

Throughout this paper we use the following notation. We denote by $\widehat{f}$ or $\mathcal{F} f$ the Fourier transform of $f$. We denote $J^{s}=(I-\Delta)^{s / 2}$ and $D^{s}=(-\Delta)^{s / 2}$. $H^{s}$ is the Sobolev space whose norm is defined by $\|f\|_{H^{s}}=\left\|J^{s} f\right\|_{L^{2}}$, and $\dot{H}^{s}$ is the homogeneous Sobolev space whose semi-norm is defined by $\|f\|_{\dot{H}^{s}}=$ $\left\|D^{s} f\right\|_{L^{2}}$. The function space $L^{p}\left(0, T ; L^{q}\left(\mathbf{R}^{2}\right)\right)$ is simply described by $L_{T}^{p} L_{x}^{q}$, and $L^{\infty}\left(0, T ; H^{s}\left(\mathbf{R}^{2}\right)\right)$ is also $L_{T}^{\infty} H_{x}^{s}$. We also denote $U(t)=e^{i t \Delta}$.

This paper is organized as follows. In section 2 , we prepare the basic estimates. In section 3, we show a priori estimates of the solution and give the proof of Theorem 1.1. In section 4, we give the proof of Theorem 1.3.

Acknowledgement. The author would like to thank Professor Yoshio Tsutsumi for valuable advice and encouragement.

\section{Linear estimates and the energy estimate}

In this section, we prepare the linear estimates and the energy estimate which we use for the proof of Theorems 1.1, 1.3. We first state the Strichartz estimates. For the proof, see [2] for example. 
Lemma 2.1. Let $n=2$. We assume $2<p \leq \infty, 2 \leq q<\infty$, and $1 / p=$ $1 / 2-1 / q$. Then, the following estimates hold.

$$
\begin{gathered}
\|U(t) f\|_{L_{T}^{p} L_{x}^{q}} \lesssim\|f\|_{L^{2}}, \\
\left\|\int_{0}^{t} U\left(t-t^{\prime}\right) F\left(t^{\prime}\right) d t^{\prime}\right\|_{L_{T}^{p} L_{x}^{q}} \lesssim\|F\|_{L_{T}^{1} L_{x}^{2}}
\end{gathered}
$$

Lemma 2.2. For $s>0,1<p<\infty$, we have

$$
\begin{aligned}
\left\|D^{s}(f g)\right\|_{L^{p}} & \lesssim\|f\|_{L^{p_{1}}}\left\|D^{s} g\right\|_{L^{p_{2}}}+\|g\|_{L^{r_{1}}}\left\|D^{s} f\right\|_{L^{r_{2}}}, \\
\left\|J^{s}(f g)\right\|_{L^{p}} & \lesssim\|f\|_{L^{p_{1}}}\left\|J^{s} g\right\|_{L^{p_{2}}}+\|g\|_{L^{r_{1}}}\left\|J^{s} f\right\|_{L^{r_{2}}},
\end{aligned}
$$

where $1 / p=1 / p_{1}+1 / p_{2}=1 / r_{1}+1 / r_{2}$ with $p<p_{1}, r_{1} \leq \infty$.

Proof. See [3, Proposition 1.2], for example. Note that $\|f\|_{F_{p, 2}^{s}} \simeq\left\|D^{s} f\right\|_{L^{p}}$ and $\|f\|_{F_{p, 2}^{s}} \simeq\left\|J^{s} f\right\|_{L^{p}}$ for $1<p<\infty$.

The Hardy-Littlewood-Sobolev inequality below is used to estimate the potential term of the equation. For the proof, see $[15, \mathrm{~V} . \S 1]$ for example.

Lemma 2.3. Let $n=2$. For $0<\alpha<2$, we define the operator $I_{\alpha}$ by

$$
I_{\alpha} f(x)=\int_{\mathbf{R}^{2}} \frac{f(y)}{|x-y|^{2-\alpha}} d y .
$$

If $1 / q=1 / p-\alpha / 2,1<p<2 / \alpha$, then we have

$$
\left\|I_{\alpha} f\right\|_{L^{q}\left(\mathbf{R}^{2}\right)} \lesssim\|f\|_{L^{p}\left(\mathbf{R}^{2}\right)} .
$$

Below we collect the estimates on the potential term $A$ defined by (1.1).

Lemma 2.4. We assume $s \geq 0,1>\delta>2 / q>0$, and $2 \leq p<\infty$ if $s>0$; $2<p<\infty$ if $s=0$. Then, we have

$$
\begin{aligned}
& \|\nabla A\|_{\dot{H}^{s}} \lesssim\|u\|_{L^{\infty}}\|u\|_{\dot{H}^{s}}, \\
& \|\nabla A\|_{L^{\infty}} \lesssim\|u\|_{L^{\infty}}\left\|J^{\delta} u\right\|_{L^{q}}, \\
& \|A\|_{L^{\infty}} \lesssim\|u\|_{L^{2}}\left\|J^{\delta} u\right\|_{L^{q}}, \\
& \left\|D^{s} A\right\|_{L^{p}} \lesssim\|u\|_{L^{p}}\|u\|_{\dot{H}^{s}} .
\end{aligned}
$$

Proof. We first notice that

$$
\partial_{k} A_{j}=C R_{k} R_{j^{\prime}} \operatorname{Im}\left(u_{1} \bar{u}_{2}\right),
$$

where $j^{\prime}=2$ if $j=1$, and $j^{\prime}=1$ if $j=2$. This is a consequence of the fact that

$$
\mathcal{F}\left[\partial_{k} A_{j}\right]=C \xi_{k} \widehat{G_{j}}(\xi) \mathcal{F}\left[\operatorname{Im}\left(u_{1} \bar{u}_{2}\right)\right],
$$

and $\widehat{G_{j}}(\xi)=C \xi_{j^{\prime}} /|\xi|^{2}$, since the Riesz transforms $R_{j}$ is defined by $\widehat{R_{j} f}=$ $-i \xi_{j} /|\xi| \widehat{f}$. For the Fourier transform of $G_{j}$, see [15, III. $\left.\S 3\right]$ for example. 
Using (2.7) and Lemma 2.2 it is easy to see that

$$
\begin{aligned}
\|\nabla A\|_{\dot{H}^{s}} & \lesssim \sum_{i, j=1}^{2}\left\|R_{i} R_{j} D^{s} \operatorname{Im}\left(u_{1} \bar{u}_{2}\right)\right\|_{L^{2}} \\
& \lesssim\left\|D^{s} \operatorname{Im}\left(u_{1} \bar{u}_{2}\right)\right\|_{L^{2}} \lesssim\|u\|_{L^{\infty}}\|u\|_{\dot{H}^{s}}
\end{aligned}
$$

since the Riesz transforms are bounded on $L^{p}$ for $1<p<\infty$. Similarly, using (2.7) and the Sobolev embedding, we have

$$
\begin{aligned}
\|\nabla A\|_{L^{\infty}} & \lesssim \sum_{i, j=1}^{2}\left\|J^{\delta} R_{i} R_{j} \operatorname{Im}\left(u_{1} \bar{u}_{2}\right)\right\|_{L^{q}} \\
& \lesssim\left\|J^{\delta} \operatorname{Im}\left(u_{1} \bar{u}_{2}\right)\right\|_{L^{q}} \lesssim\|u\|_{L^{\infty}}\left\|J^{\delta} u\right\|_{L^{q}},
\end{aligned}
$$

where $\delta>2 / q$, and we applied Lemma 2.2 for the last inequality. For the proof of $(2.5)$ we use Lemma 2.3. In fact, since $\left|G_{j}(x)\right| \lesssim 1 /|x|$, we have

$$
\|A\|_{L^{\infty}} \lesssim\left\|J^{\delta} A\right\|_{L^{q}} \lesssim\left\|I_{1}\left|J^{\delta} \operatorname{Im}\left(u_{1} \bar{u}_{2}\right)\right|\right\|_{L^{q}} .
$$

Since $q>2$, we are able to apply Lemma 2.3 for $1 / q=1 / r-1 / 2$ to obtain

$$
\left\|I_{1}\left|J^{\delta} \operatorname{Im}\left(u_{1} \bar{u}_{2}\right)\right|\right\|_{L^{q}} \lesssim\left\|J^{\delta} \operatorname{Im}\left(u_{1} \bar{u}_{2}\right)\right\|_{L^{r}} \lesssim\|u\|_{L^{2}}\left\|J^{\delta} u\right\|_{L^{q}}
$$

The proof of (2.6) are divided into three cases. The case $s=0$ is similar to (2.5). In fact, we have

$$
\|A\|_{L^{p}} \lesssim\left\|I_{1}|u|^{2}\right\|_{L^{p}} \lesssim\left\||u|^{2}\right\|_{L^{r}} \lesssim\|u\|_{L^{p}}\|u\|_{L^{2}}
$$

where $r$ is determined by $1 / p=1 / r-1 / 2$ with $2<p<\infty$. We next consider the case $0<s<1$. In this case, we first notice that

$$
\mathcal{F}\left[D^{s} A_{j}\right]=C|\xi|^{s} \widehat{G}_{j}(\xi) \mathcal{F}\left[\operatorname{Im}\left(u_{1} \bar{u}_{2}\right)\right]=C \frac{\xi_{j^{\prime}}}{|\xi|^{2-s}} \mathcal{F}\left[\operatorname{Im}\left(u_{1} \bar{u}_{2}\right)\right] .
$$

Since $\mathcal{F}^{-1}\left[\xi_{j^{\prime}} /|\xi|^{2-s}\right]=C x_{j^{\prime}} /|x|^{2+s}$ (see [15, III. $\left.\S 3\right]$ for example), we have

$$
\left\|D^{s} A\right\|_{L^{p}} \lesssim\left\|I_{1-s}|u|^{2}\right\|_{L^{p}} \lesssim\left\||u|^{2}\right\|_{L^{r}} \lesssim\|u\|_{L^{p}}\|u\|_{L^{2 /(1-s)}},
$$

where $r$ is determined by $1 / p=1 / r-(1-s) / 2$ with $2 \leq p<\infty$. Then, the Sobolev embedding $\dot{H}^{s}\left(\mathbf{R}^{2}\right) \hookrightarrow L^{2 /(1-s)}\left(\mathbf{R}^{2}\right)$ (note that $u \in L^{p}$ ) enables us to obtain (2.6). In the case where $s \geq 1$, we observe that from $(2.8) \mathcal{F}\left[D^{s} A_{j}\right]$ is written as

$$
\mathcal{F}\left[D^{s} A_{j}\right]=C \frac{\xi_{j^{\prime}}}{|\xi|^{2-\varepsilon}}|\xi|^{s-\varepsilon} \mathcal{F}\left[\operatorname{Im}\left(u_{1} \bar{u}_{2}\right)\right]
$$

for $0<\varepsilon<1$. Thus, similarly as above, we obtain

$$
\begin{aligned}
\left\|D^{s} A\right\|_{L^{p}} & \lesssim\left\|I_{1-\varepsilon}\left|D^{s-\varepsilon} \operatorname{Im}\left(u_{1} \bar{u}_{2}\right)\right|\right\|_{L^{p}} \\
& \lesssim\left\|D^{s-\varepsilon} \operatorname{Im}\left(u_{1} \bar{u}_{2}\right)\right\|_{L^{r}} \\
& \lesssim\|u\|_{L^{p}}\left\|D^{s-\varepsilon} u\right\|_{L^{2 /(1-\varepsilon)}} \lesssim\|u\|_{L^{p}}\left\|D^{s} u\right\|_{L^{2}},
\end{aligned}
$$

where $r$ is determined by $1 / p=1 / r-(1-\varepsilon) / 2$ with $2 \leq p<\infty$. 
We next prove the energy estimate for the solution to the modified Schrödinger map.

Proposition 2.5. Let $s \geq 0$ and let $u$ be a solution to $(M S M)$ on $(0, T) \times \mathbf{R}^{2}$. Then, for $T>0$, we have

$$
\begin{gathered}
\|u\|_{L_{T}^{\infty} L_{x}^{2}}=\left\|u_{0}\right\|_{L^{2}}, \\
\|u\|_{L_{T}^{\infty} H_{x}^{s}} \leq\left\|u_{0}\right\|_{H^{s}} \exp \left\{C\left(1+\left\|u_{0}\right\|_{L^{2}}^{2}\right) T^{2 / q}\left\|J^{\delta} u\right\|_{L_{T}^{p} L_{x}^{q}}^{2}\right\},
\end{gathered}
$$

where $\delta>2 / q>0,1 / p=1 / 2-1 / q$.

Proof. The conservation of the $L^{2}$-norm (2.9) is easily obtained from the equation (MSM). In fact, multiplying the first equation in (MSM) by $\bar{u}_{1}$ and integrating over $\mathbf{R}^{n}$, and then taking the imaginary part, we obtain

$$
\partial_{t}\left\|u_{1}(t)\right\|_{L^{2}}^{2}=2 \int \operatorname{Im}\left(u_{2} \bar{u}_{1}\right) \operatorname{Re}\left(u_{2} \bar{u}_{1}\right) d x
$$

since $\operatorname{div} A=0$, and $B$ is real valued. Similarly, we obtain

$$
\begin{aligned}
\partial_{t}\left\|u_{2}(t)\right\|_{L^{2}}^{2} & =2 \int \operatorname{Im}\left(u_{1} \bar{u}_{2}\right) \operatorname{Re}\left(u_{1} \bar{u}_{2}\right) d x \\
& =-2 \int \operatorname{Im}\left(u_{2} \bar{u}_{1}\right) \operatorname{Re}\left(u_{2} \bar{u}_{1}\right) d x .
\end{aligned}
$$

Thus, we obtain $\partial_{t}\|u(t)\|_{L^{2}}^{2}=\partial_{t}\left\|u_{1}(t)\right\|_{L^{2}}^{2}+\partial_{t}\left\|u_{2}(t)\right\|_{L^{2}}^{2}=0$ which implies (2.9).

For the proof of the energy estimate (2.10), we employ [12, Propositions 2] which states

$$
\begin{aligned}
\partial_{t}\|u(t)\|_{\dot{H}^{s}}^{2} \lesssim & \left(\|\nabla A(t)\|_{\dot{H}^{s}}\|u(t)\|_{L^{\infty}}+\|\nabla A(t)\|_{L^{\infty}}\|u(t)\|_{\dot{H}^{s}}+\|B(t) u(t)\|_{\dot{H}^{s}}\right. \\
& \left.+\left\|\left.|| A(t)\right|^{2} u(t)\right\|_{\dot{H}^{s}}+\left\|\operatorname{Im}\left(u_{1}(t) \bar{u}_{2}(t)\right) u(t)\right\|_{\dot{H}^{s}}\right)\|u(t)\|_{\dot{H}^{s}} .
\end{aligned}
$$

In what follows, we estimate each term on the right hand side of (2.11) to obtain (2.10) assuming $s>0$.

From (2.3) we have

$$
\|\nabla A(t)\|_{\dot{H}^{s}}\|u(t)\|_{L^{\infty}} \lesssim\|u(t)\|_{L^{\infty}}^{2}\|u(t)\|_{\dot{H}^{s}} \lesssim\left\|J^{\delta} u(t)\right\|_{L^{q}}^{2}\|u(t)\|_{\dot{H}^{s}}
$$

by the Sobolev embedding, where $\delta>2 / q$. Similarly, from (2.4) we have

$$
\begin{aligned}
\|\nabla A(t)\|_{L^{\infty}}\|u(t)\|_{\dot{H}^{s}} & \lesssim\|u(t)\|_{L^{\infty}}\left\|J^{\delta} u(t)\right\|_{L^{q}}\|u(t)\|_{\dot{H}^{s}} \\
& \lesssim\left\|J^{\delta} u(t)\right\|_{L^{q}}^{2}\|u(t)\|_{\dot{H}^{s}} .
\end{aligned}
$$

From the definition (1.3) we have

$$
\|B(t) u(t)\|_{\dot{H}^{s}} \lesssim \sum_{j, k=1}^{2} \|\left(R_{j} R_{k} \operatorname{Re}\left(u_{j}(t) \bar{u}_{k}(t)\right) u(t)\left\|_{\dot{H}^{s}}+\right\||u(t)|^{2} u(t) \|_{\dot{H}^{s}} .\right.
$$


As for the first term on the right hand side of (2.14), we apply Lemma 2.2 to get

$$
\begin{aligned}
& \left\|D^{s}\left(R_{j} R_{k} \operatorname{Re}\left(u_{j} \bar{u}_{k}\right) u\right)\right\|_{L^{2}} \\
& \lesssim\|u\|_{L^{\infty}}\left\|D^{s} R_{j} R_{k} \operatorname{Re}\left(u_{j} \bar{u}_{k}\right)\right\|_{L^{2}}+\left\|R_{j} R_{k} \operatorname{Re}\left(u_{j} \bar{u}_{k}\right)\right\|_{L^{\infty}}\left\|D^{s} u\right\|_{L^{2}} \\
& \lesssim\|u\|_{L^{\infty}}\left\|D^{s} \operatorname{Re}\left(u_{j} \bar{u}_{k}\right)\right\|_{L^{2}}+\left\|J^{\delta} R_{j} R_{k} \operatorname{Re}\left(u_{j} \bar{u}_{k}\right)\right\|_{L^{q}}\left\|D^{s} u\right\|_{L^{2}} \\
& \lesssim\|u\|_{L^{\infty}}^{2}\left\|D^{s} u\right\|_{L^{2}}+\left\|J^{\delta} \operatorname{Re}\left(u_{j} \bar{u}_{k}\right)\right\|_{L^{q}}\left\|D^{s} u\right\|_{L^{2}} \\
& \lesssim\|u\|_{L^{\infty}}^{2}\left\|D^{s} u\right\|_{L^{2}}+\|u\|_{L^{\infty}}\left\|J^{\delta} u\right\|_{L^{q}}\left\|D^{s} u\right\|_{L^{2}} .
\end{aligned}
$$

Meanwhile, the following estimate of the second term on the right hand side of $(2.14)$

$$
\left\|D^{s}\left(|u|^{2} u\right)\right\|_{L^{2}} \lesssim\|u\|_{L^{\infty}}^{2}\left\|D^{s} u\right\|_{L^{2}}
$$

is also derived by applying Lemma 2.2 repeatedly. Thus, combining estimates above we obtain

$$
\|B(t) u(t)\|_{\dot{H}^{s}} \lesssim\left\|J^{\delta} u(t)\right\|_{L^{q}}^{2}\|u(t)\|_{\dot{H}^{s}} .
$$

Here, we notice that the following estimate of the fifth term on the right hand side of (2.11)

$$
\left\|\operatorname{Im}\left(u_{1}(t) \bar{u}_{2}(t)\right) u(t)\right\|_{\dot{H}^{s}} \lesssim\|u(t)\|_{L^{\infty}}^{2}\left\|D^{s} u(t)\right\|_{L^{2}}
$$

is similarly obtained as (2.15). Finally we estimate the fourth term on the right hand side of (2.11). Applying (2.5) and (2.6) we have

$$
\begin{aligned}
\left\|D^{s}\left(|A|^{2} u\right)\right\|_{L^{2}} & \lesssim\|u\|_{L^{\infty}}\left\|D^{s}|A|^{2}\right\|_{L^{2}}+\|A\|_{L^{\infty}}^{2}\left\|D^{s} u\right\|_{L^{2}} \\
& \lesssim\|u\|_{L^{\infty}}\|A\|_{L^{\infty}}\left\|D^{s} A\right\|_{L^{2}}+\|A\|_{L^{\infty}}^{2}\left\|D^{s} u\right\|_{L^{2}} \\
& \lesssim\|u\|_{L^{2}}^{2}\|u\|_{L^{\infty}}\left\|J^{\delta} u\right\|_{L^{q}}\|u\|_{\dot{H}^{s}}+\|u\|_{L^{2}}^{2}\left\|J^{\delta} u\right\|_{L^{q}}^{2}\|u\|_{\dot{H}^{s}} \\
& \lesssim\left\|u_{0}\right\|_{L^{2}}^{2}\left\|J^{\delta} u\right\|_{L^{q}}^{2}\|u\|_{\dot{H}^{s}}
\end{aligned}
$$

Combining (2.11), (2.12), (2.13) (2.16), (2.17), and (2.18), we obtain

$$
\partial_{t}\|u(t)\|_{\dot{H}^{s}}^{2} \leq C\left(1+\left\|u_{0}\right\|_{L^{2}}^{2}\right)\left\|J^{\delta} u(t)\right\|_{L^{q}}^{2}\|u(t)\|_{\dot{H}^{s}}^{2} .
$$

Thus, applying the Gronwall inequality we have

$$
\|u(t)\|_{\dot{H}^{s}} \leq\left\|u_{0}\right\|_{\dot{H}^{s}} \exp \left(C\left(1+\left\|u_{0}\right\|_{L^{2}}^{2}\right) \int_{0}^{T}\left\|J^{\delta} u\left(t^{\prime}\right)\right\|_{L^{q}}^{2} d t^{\prime}\right) .
$$

Therefore, (2.9) and the Hölder inequality with respect to the time integral enables us to obtain (2.10).

We finally state the crucial estimate for the proof of Theorem 1.1. This type of estimate was first given by Koch-Tzvetkov [10] and refined by Kenig-Koenig [8] in the context of the Benjamin-Ono equation. 
Proposition 2.6. Let $T \leq 1$. We assume that $w$ is a solution to the equation

$$
i \partial_{t} w+\Delta w=F_{1}+F_{2}, \quad(t, x) \in(0, T) \times \mathbf{R}^{2} .
$$

Then, for $s \in \mathbf{R}, \varepsilon^{\prime}>0$, we have

$$
\left\|J^{s} w\right\|_{L_{T}^{p} L_{x}^{q}} \lesssim\|w\|_{L_{T}^{\infty} H^{s+1 / 2+\varepsilon^{\prime}}}+\left\|F_{1}\right\|_{L_{T}^{2} H_{x}^{s-1 / 2}}+\left\|F_{2}\right\|_{L_{T}^{1} H_{x}^{s}},
$$

where $1 / p=1 / 2-1 / q, 2 \leq q<\infty$.

Proof. The proof is essentially due to [8, Proposition 2.8]. We first introduce the Littlewood-Paley decomposition. Let $\varphi \in C_{0}^{\infty}\left(\mathbf{R}^{2}\right)$ satisfy $\varphi(\xi)=1$ for $|\xi| \leq 1 / 2$, $\varphi(\xi)=0$ for $|\xi| \geq 1$. And we set $\eta(\xi)=\varphi(\xi / 2)-\varphi(\xi)$ and set $\eta_{k}(\xi)=\eta\left(\xi / 2^{k}\right)$ for $k \geq 0$ so that $\operatorname{supp} \eta_{k} \subset\left\{2^{k-1} \leq|\xi| \leq 2^{k+1}\right\}$ and $1=\varphi(\xi)+\sum_{k=0}^{\infty} \eta_{k}(\xi)$. Then, we define $\Delta_{k}$ by $\widehat{\Delta_{k} f}=\eta_{k} \widehat{f}$ and $S_{0}$ by $\widehat{S_{0} f}=\varphi \widehat{f}$, where $\widehat{g}$ denotes the Fourier transform of $g$. Using the notation above, it is known that

$$
\|f\|_{L^{r}\left(\mathbf{R}^{n}\right)} \simeq\left\|\left(\left|S_{0} f\right|^{2}+\sum_{k=0}^{\infty}\left|\Delta_{k} f\right|^{2}\right)^{1 / 2}\right\|_{L^{r}\left(\mathbf{R}^{n}\right)}
$$

holds for $1<r<\infty$.

We next prepare the disjoint decomposition of the time interval $[0, T]=$ $\cup_{j=1}^{m} I_{j}$, where $I_{j}=\left[a_{j}, a_{j+1}\right)$ satisfy $\left|I_{j}\right|=2^{-j}$ for $1 \leq j \leq m-1$ and $2^{-j} \leq\left|I_{m}\right| \leq 2^{-j+1}$. Then, we have $m \leq 2^{j}$, since $(m-1) 2^{-j}+\left|I_{m}\right|=T$ implies $2^{-j} m \leq T-2^{-j}+2^{-j}=T \leq 1$.

Since $2 \leq q<\infty$, using the Littlewood-Paley decomposition of $J^{s} w$ we have

$$
\begin{aligned}
\left\|J^{s} w\right\|_{L_{T}^{p} L_{x}^{q}} & \lesssim\left\|\left(\left|S_{0} J^{s} w\right|^{2}+\sum_{k=0}^{\infty}\left|\Delta_{k} J^{s} w\right|^{2}\right)^{1 / 2}\right\|_{L_{T}^{p} L_{x}^{q}} \\
& \lesssim\left(\left\|J^{s} S_{0} w\right\|_{L_{T}^{p} L_{x}^{q}}^{2}+\sum_{k=0}^{\infty}\left\|J^{s} \Delta_{k} w\right\|_{L_{T}^{p} L_{x}^{q}}^{2}\right)^{1 / 2} .
\end{aligned}
$$

For the last inequality above we used Minkowski's integral inequality, since $p, q \geq 2$. Before applying Strichartz estimates, we use the decomposition $[0, T]=$ $\cup_{j=1}^{m} I_{j}$ to estimate $J^{s} \Delta_{k} w$ as follows.

$$
\begin{aligned}
\left\|J^{s} \Delta_{k} w\right\|_{L_{T}^{p} L_{x}^{q}} & \lesssim 2^{s k}\left\|\Delta_{k} w\right\|_{L_{T}^{p} L_{x}^{q}} \\
& =2^{s k}\left(\sum_{j=1}^{m}\left\|\Delta_{k} w\right\|_{L_{I_{j}}^{p} L_{x}^{q}}^{p}\right)^{1 / p} \\
& \lesssim 2^{s k}\left(\sum_{j=1}^{m}\left\|\Delta_{k} w\right\|_{L_{I_{j}}^{p} L_{x}^{q}}^{2}\right)^{1 / 2},
\end{aligned}
$$

since $l^{2} \hookrightarrow l^{p}$ for $p>2$. Since $\Delta_{k} w$ satisfies the following integral equation

$$
\Delta_{k} w(t)=U(t) \Delta_{k} w\left(a_{j}\right)-i \int_{a_{j}}^{t} U\left(t-t^{\prime}\right) \Delta_{k}\left(F_{1}\left(t^{\prime}\right)+F_{2}\left(t^{\prime}\right)\right) d t^{\prime}
$$


for $t \in I_{j}$, applying Lemma 2.1 we have

$$
\left\|\Delta_{k} w\right\|_{L_{I_{j}}^{p} L_{x}^{q}} \lesssim\left\|\Delta_{k} w\left(a_{j}\right)\right\|_{L^{2}}^{2}+\left\|\Delta_{k} F_{1}\right\|_{L_{I_{j}}^{1} L_{x}^{2}}+\left\|\Delta_{k} F_{2}\right\|_{L_{I_{j}}^{1} L_{x}^{2}}
$$

Thus, we obtain

$$
\begin{aligned}
& 2^{s k}\left(\sum_{j=1}^{m}\left\|\Delta_{k} w\right\|_{L_{I_{j}}^{p} L_{x}^{q}}^{2}\right)^{1 / 2} \\
\lesssim & 2^{s k}\left\{\sum_{j=1}^{m}\left(\left\|\Delta_{k} w\left(a_{j}\right)\right\|_{L^{2}}^{2}+\left\|\Delta_{k} F_{1}\right\|_{L_{I_{j}}^{1} L_{x}^{2}}^{2}+\left\|\Delta_{k} F_{2}\right\|_{L_{I_{j}}^{1} L_{x}^{2}}^{2}\right)\right\}^{1 / 2} \\
\lesssim & 2^{s k}\left\{m\left\|\Delta_{k} w\right\|_{L_{T}^{\infty} L_{x}^{2}}^{2}+\sum_{j=1}^{m}\left|I_{j}\right|\left\|\Delta_{k} F_{1}\right\|_{L_{I_{j}}^{2} L_{x}^{2}}^{2}\right\}^{1 / 2}+2^{s k}\left\|\Delta_{k} F_{2}\right\|_{L_{T}^{1} L_{x}^{2}} \\
\lesssim & 2^{(s+1 / 2) k}\left\|\Delta_{k} w\right\|_{L_{T}^{\infty} L_{x}^{2}}+2^{(s-1 / 2) k}\left\|\Delta_{k} F_{1}\right\|_{L_{T}^{2} L_{x}^{2}}+2^{s k}\left\|\Delta_{k} F_{2}\right\|_{L_{T}^{1} L_{x}^{2}} \\
\lesssim & 2^{-\varepsilon^{\prime} k}\|w\|_{L_{T}^{\infty} H_{x}^{s+1 / 2+\varepsilon^{\prime}}}+\left\|\Delta_{k} J^{s-1 / 2} F_{1}\right\|_{L_{T}^{2} L_{x}^{2}}+\left\|\Delta_{k} J^{s} F_{2}\right\|_{L_{T}^{1} L_{x}^{2}} .
\end{aligned}
$$

For the last term in the second inequality above, we used the triangle inequality in $l^{2}$ and then applied $l^{1} \hookrightarrow l^{2}$. Combining the estimates above we obtain

$$
\begin{aligned}
& \left\|J^{s} \Delta_{k} w\right\|_{L_{T}^{p} L_{x}^{q}} \\
& \lesssim 2^{-\varepsilon^{\prime} k}\|w\|_{L_{T}^{\infty} H_{x}^{s+1 / 2+\varepsilon^{\prime}}}+\left\|\Delta_{k} J^{s-1 / 2} F_{1}\right\|_{L_{T}^{2} L_{x}^{2}}+\left\|\Delta_{k} J^{s} F_{2}\right\|_{L_{T}^{1} L_{x}^{2}} .
\end{aligned}
$$

Meanwhile, applying Lemma 2.1 it is easy to see that

$$
\begin{aligned}
\left\|J^{s} S_{0} w\right\|_{L_{T}^{p} L_{x}^{q}} & \lesssim\left\|S_{0} w(0)\right\|_{L^{2}}+\left\|S_{0} F_{1}\right\|_{L_{T}^{1} L_{x}^{2}}+\left\|S_{0} F_{2}\right\|_{L_{T}^{1} L_{x}^{2}} \\
& \lesssim\|w\|_{L_{T}^{\infty} L_{x}^{2}}+\left\|S_{0} J^{s-1 / 2} F_{1}\right\|_{L_{T}^{2} L_{x}^{2}}+\left\|S_{0} J^{s} F_{2}\right\|_{L_{T}^{1} L_{x}^{2}}
\end{aligned}
$$


Therefore, we obtain

$$
\begin{aligned}
\left(\left\|J^{s} S_{0} w\right\|_{L_{T}^{p} L_{x}^{q}}^{2}+\right. & \left.\sum_{k=0}^{\infty}\left\|J^{s} \Delta_{k} w\right\|_{L_{T}^{p} L_{x}^{q}}^{2}\right)^{1 / 2} \\
\lesssim & \left(\sum_{k=0}^{\infty} 2^{-2 \varepsilon^{\prime} k}\|w\|_{L_{T}^{\infty} H_{x}^{s+1 / 2+\varepsilon^{\prime}}}^{2}\right)^{1 / 2} \\
& +\left(\left\|S_{0} J^{s-1 / 2} F_{1}\right\|_{L_{T}^{2} L_{x}^{2}}^{2}+\sum_{k=0}^{\infty}\left\|\Delta_{k} J^{s-1 / 2} F_{1}\right\|_{L_{T}^{2} L_{x}^{2}}^{2}\right)^{1 / 2} \\
& +\left(\left\|S_{0} J^{s} F_{2}\right\|_{L_{T}^{1} L_{x}^{2}}^{2}+\sum_{k=0}^{\infty}\left\|\Delta_{k} J^{s} F_{2}\right\|_{L_{T}^{1} L_{x}^{2}}^{2}\right)^{1 / 2} \\
\lesssim & \left(\sum_{k=0}^{\infty} 2^{-2 \varepsilon^{\prime} k}\right)^{1 / 2}\|w\|_{L_{T}^{\infty} H_{x}^{s+1 / 2+\varepsilon^{\prime}}} \\
& +\left\|\left(\left|S_{0} J^{s-1 / 2} F_{1}\right|^{2}+\sum_{k=0}^{\infty}\left|\Delta_{k} J^{s-1 / 2} F_{1}\right|^{2}\right)^{1 / 2}\right\|_{L_{T}^{2} L_{x}^{2}} \\
& +\left\|\left(\left|S_{0} J^{s} F_{2}\right|^{2}+\sum_{k=0}^{\infty}\left|\Delta_{k} J^{s} F_{2}\right|^{2}\right)^{1 / 2}\right\|_{L_{T}^{1} L_{x}^{2}} \\
\lesssim & \|w\| L_{L_{T}^{\infty} H_{x}^{s+1 / 2+\varepsilon^{\prime}}}+\left\|J^{s-1 / 2} F_{1}\right\|_{L_{T}^{2} L_{x}^{2}}+\left\|J^{s} F_{2}\right\|_{L_{T}^{1} L_{x}^{2}}
\end{aligned}
$$

where we applied Minkowski's integral inequality again for the second inequality above. This completes the proof of Proposition 2.6.

\section{A priori estimate}

In this section, we prove a priori estimate for the solution to the modified Schrödinger map applying the estimates in the preceding section. Then, using the a priori estimate, we give the proof of Theorem 1.1.

Theorem 3.1. Let $u_{0} \in H^{\infty}\left(\mathbf{R}^{2}\right)$ and let $u$ be a solution to (MSM). Then, for any $\varepsilon>0$, there exists $T$ satisfying

$$
\min \left\{1, C /\left(\left(1+\left\|u_{0}\right\|_{L^{2}}^{q}\right)\left\|u_{0}\right\|_{H^{1 / 2+\varepsilon}}^{q}\right)\right\} \leq T \leq 1
$$

and $M>0$ such that

$$
\left\|J^{\delta} u\right\|_{L_{T}^{p} L_{x}^{q}} \leq M\left\|u_{0}\right\|_{H^{1 / 2+\varepsilon}}
$$

where $\varepsilon>\delta>2 / q>0,1 / p=1 / 2-1 / q$.

Proof. Applying Proposition 2.6 for $s=\delta, F_{1}=-2 i A \cdot \nabla u_{j}$, and $F_{2}=B u_{j}+$ $|A|^{2} u_{j} \pm \operatorname{Im}\left(u_{2} \bar{u}_{1}\right) u_{j}$, we obtain

$$
\begin{aligned}
\left\|J^{\delta} u\right\|_{L_{T}^{p} L_{x}^{q}} \lesssim & \|u\|_{L_{T}^{\infty} H_{x}^{1 / 2+\varepsilon}}+\|A \cdot \nabla u\|_{L_{T}^{2} H_{x}^{-1 / 2+\delta}} \\
& +\|B u\|_{L_{T}^{1} H_{x}^{\delta}}+\left\||A|^{2} u\right\|_{L_{T}^{1} H_{x}^{\delta}}+\left\|\operatorname{Im}\left(u_{2} \bar{u}_{1}\right) u\right\|_{L_{T}^{1} H_{x}^{\delta}}
\end{aligned}
$$


where we substituted $\delta+\varepsilon^{\prime}=\varepsilon$. In what follows we estimate each term on the right hand side of (3.2) to obtain (3.1).

The first term is easily estimated by applying energy estimate (2.10) directly,

$$
\|u\|_{L_{T}^{\infty} H_{x}^{1 / 2+\varepsilon}} \leq\left\|u_{0}\right\|_{H^{1 / 2+\varepsilon}} \exp \left\{C\left(1+\left\|u_{0}\right\|_{L^{2}}^{2}\right) T^{2 / q}\left\|J^{\delta} u\right\|_{L_{T}^{p} L_{x}^{q}}^{2}\right\} .
$$

As for the second term we notice that $A \cdot \nabla u=\operatorname{div}(A u)$, since $\operatorname{div} A=0$. Using this, we have

$$
\begin{aligned}
\|A \cdot \nabla u\|_{L_{T}^{2} H_{x}^{-1 / 2+\delta}} & \lesssim\left\|J^{-1 / 2+\delta} \nabla(A u)\right\|_{L_{T}^{2} L_{x}^{2}} \\
\lesssim & \lesssim\left\|D^{1 / 2+\delta}(A u)\right\|_{L_{T}^{2} L_{x}^{2}}+\|A u\|_{L_{T}^{2} L_{x}^{2}} \\
& \lesssim\|u\|_{L_{T}^{2} L_{x}^{\infty}}\left\|D^{1 / 2+\delta} A\right\|_{L_{T}^{\infty} L_{x}^{2}} \\
& +\|A\|_{L_{T}^{2} L_{x}^{\infty}}\left(\left\|D^{1 / 2+\delta} u\right\|_{L_{T}^{\infty} L_{x}^{2}}+\|u\|_{L_{T}^{\infty} L_{x}^{2}}\right) .
\end{aligned}
$$

Then, applying (2.5), (2.6), we have

$$
\begin{gathered}
\left\|D^{1 / 2+\delta} A\right\|_{L_{T}^{\infty} L_{x}^{2}} \lesssim\|u\|_{L_{T}^{\infty} L_{x}^{2}}\left\|D^{1 / 2+\delta} u\right\|_{L_{T}^{\infty} L_{x}^{2}}, \\
\|A\|_{L_{T}^{2} L_{x}^{\infty}} \lesssim\|u\|_{L_{T}^{\infty} L_{x}^{2}}\left\|J^{\delta} u\right\|_{L_{T}^{2} L_{x}^{q} .}
\end{gathered}
$$

Thus, combining (2.9) and the energy estimate (2.10), we obtain

$$
\begin{aligned}
\|A \cdot \nabla u\|_{L_{T}^{2} H_{x}^{-1 / 2+\delta}} & \lesssim\|u\|_{L_{T}^{\infty} L_{x}^{2}}\left\|J^{\delta} u\right\|_{L_{T}^{2} L_{x}^{q}}\|u\|_{L_{T}^{\infty} H^{1 / 2+\delta}} \\
& \lesssim\left(1+\left\|u_{0}\right\|_{L^{2}}^{2}\left\|J^{\delta} u\right\|_{L_{T}^{2} L_{x}^{q}}^{2}\|u\|_{L_{T}^{\infty} H^{1 / 2+\delta}}\right. \\
& \lesssim\left\|u_{0}\right\|_{H^{1 / 2+\delta}} \exp \left\{C\left(1+\left\|u_{0}\right\|_{L^{2}}^{2}\right) T^{2 / q}\left\|J^{\delta} u\right\|_{L_{T}^{p} L_{x}^{q}}^{2}\right\} .
\end{aligned}
$$

As for the third and the fifth term, we are able to use (2.16), (2.17) to obtain

$$
\begin{aligned}
& \|B u\|_{L_{T}^{1} H_{x}^{\delta}}+\left\|\operatorname{Im}\left(u_{2} \bar{u}_{1}\right) u\right\|_{L_{T}^{1} H_{x}^{\delta}} \\
& \lesssim\left\|J^{\delta} u\right\|_{L_{T}^{2} L_{x}^{q}}^{2}\|u\|_{L_{T}^{\infty} H^{\delta}} \\
& \lesssim\left\|u_{0}\right\|_{H^{\delta}} \exp \left\{C\left(1+\left\|u_{0}\right\|_{L^{2}}^{2}\right) T^{2 / q}\left\|J^{\delta} u\right\|_{L_{T}^{p} L_{x}^{q}}^{2}\right\} .
\end{aligned}
$$

Similarly, we are able to use (2.18) to obtain

$$
\begin{aligned}
\left\||A|^{2} u\right\|_{L_{T}^{1} H_{x}^{\delta}} & \lesssim\left\|u_{0}\right\|_{L^{2}}^{2}\left\|J^{\delta} u\right\|_{L_{T}^{2} L_{x}^{q}}^{2}\|u\|_{L_{T}^{\infty} H^{\delta}} \\
& \lesssim\left\|u_{0}\right\|_{H^{\delta}} \exp \left\{C\left(1+\left\|u_{0}\right\|_{L^{2}}^{2}\right) T^{2 / q}\left\|J^{\delta} u\right\|_{L_{T}^{p} L_{x}^{q}}^{2}\right\} .
\end{aligned}
$$

Combining estimates above, we obtain

$$
\left\|J^{\delta} u\right\|_{L_{T}^{p} L_{x}^{q}} \leq C_{0}\left\|u_{0}\right\|_{H^{1 / 2+\varepsilon}} \exp \left\{C_{1}\left(1+\left\|u_{0}\right\|_{L^{2}}^{2}\right) T^{2 / q}\left\|J^{\delta} u\right\|_{L_{T}^{p} L_{x}^{q}}^{2}\right\} .
$$

Now we set $K(T)=\left\|J^{\delta} u\right\|_{L_{T}^{p} L_{x}^{q}}^{2}$. Then, $K(T)$ is a continuous function with respect to $T$ since $2 \leq p<\infty$, and (3.3) implies

$$
K(T) \leq C_{0}^{2}\left\|u_{0}\right\|_{H^{1 / 2+\varepsilon}}^{2} \exp \left\{2 C_{1}\left(1+\left\|u_{0}\right\|_{L^{2}}^{2}\right) T^{2 / q} K(T)\right\} .
$$


If $K(T) \leq C_{0}^{2} e\left\|u_{0}\right\|_{H^{1 / 2+\varepsilon}}^{2}$ holds for $0 \leq T \leq 1$, then the conclusion of Theorem 3.1 follows. On the other hand, in the case of there exists $T_{1} \in(0,1)$ such that $K\left(T_{1}\right)>C_{0}^{2} e\left\|u_{0}\right\|_{H^{1 / 2+\varepsilon}}^{2}$, we set

$$
T_{0}=\inf \left\{T>0 ; K(T)>C_{0}^{2} e\left\|u_{0}\right\|_{H^{1 / 2+\varepsilon}}^{2}\right\} .
$$

Then, $T_{0}>0$ and we have $K\left(T_{0}\right)=C_{0}^{2} e\left\|u_{0}\right\|_{H^{1 / 2+\varepsilon}}^{2}$. Thus, (3.4) with $T=T_{0}$ implies that

$$
e \leq \exp \left\{2 C_{1}\left(1+\left\|u_{0}\right\|_{L^{2}}^{2}\right) T_{0}^{2 / q} C_{0}^{2} e\left\|u_{0}\right\|_{H^{1 / 2+\varepsilon}}^{2}\right\} .
$$

Therefore, we obtain the lower bound of $T_{0}$,

$$
T_{0} \geq \frac{1}{\left(2 C_{0}^{2} C_{1} e\right)^{q / 2}\left(1+\left\|u_{0}\right\|_{L^{2}}^{q}\right)\left\|u_{0}\right\|_{H^{1 / 2+\varepsilon}}^{q}},
$$

and for $0 \leq T \leq T_{0}, K(T) \leq K\left(T_{0}\right)=C_{0}^{2} e\left\|u_{0}\right\|_{H^{1 / 2+\varepsilon}}^{2}$ holds. This completes the proof of Theorem 3.1.

Using Theorem 3.1, we are able to prove Theorem 1.1 as follows.

Proof of Theorem 1.1. Let $\rho \in C_{0}^{\infty}\left(\mathbf{R}^{2}\right)$ satisfy $\rho(x)=1$ if $|x| \leq 1, \rho(x)=0$ if $|x| \geq 2$. Then, for $u_{0} \in H^{s}\left(\mathbf{R}^{2}\right)$ with $s>1 / 2$ we define $\rho_{\lambda}(x)=1 / \lambda^{2} \rho(x / \lambda)$ and set $u_{0}^{\lambda}=\rho_{\lambda} * u_{0}$ and the corresponding solution $u^{\lambda}$. The existence of such solution is assured by [12, Theorem 4] (see also [1, Theorem 2.1]).

From Theorem 3.1, $u^{\lambda}$ has a priori uniform bound

$$
\left\|J^{\delta} u^{\lambda}\right\|_{L_{T}^{p} L_{x}^{q}} \leq M\left\|u_{0}^{\lambda}\right\|_{H^{1 / 2+\varepsilon}} \leq C M\left\|u_{0}\right\|_{H^{1 / 2+\varepsilon}},
$$

for $\varepsilon>\delta>2 / q>0,1 / p=1 / 2-1 / q$. Thus, by Proposition 2.5 we obtain

$$
\left\|u^{\lambda}\right\|_{L_{T}^{\infty} H_{x}^{s}} \leq C\left\|u_{0}\right\|_{H^{s}} \exp \left(C M^{2}\left(1+\left\|u_{0}\right\|_{L^{2}}^{2}\right)\left\|u_{0}\right\|_{H^{1 / 2+\varepsilon}}^{2}\right),
$$

where we notice that $\varepsilon$ can be chosen so that $s \geq 1 / 2+\varepsilon$ with $\varepsilon>\delta$. Note that we may consider the solution $u^{\lambda}$ exists on $[0, T]$ satisfying $(3.5),(3.6)$, where the lower bound of $T$ is determined independently of $\lambda$ satisfying

$$
\min \left\{1, C /\left(\left(1+\left\|u_{0}\right\|_{L^{2}}^{q}\right)\left\|u_{0}\right\|_{H^{1 / 2+\varepsilon}}^{q}\right)\right\} \leq T .
$$

Using (3.5), (3.6), and the equation (MSM), we also obtain

$$
\left\|\partial_{t} u^{\lambda}\right\|_{L_{T}^{p / 2} H_{x}^{-2}} \leq M^{\prime}
$$

Form uniform estimates on $u^{\lambda}$ above, we observe that there exist subsequence $\left\{u^{\lambda^{\prime}}\right\}$ and $u \in L_{T}^{\infty} H_{x}^{s} \cap L_{T}^{p}\left(J^{-\delta} L^{q}\right)_{x}$ such that

$$
\begin{gathered}
u^{\lambda^{\prime}} \rightarrow u \quad \text { *-weakly in } L_{T}^{\infty} H_{x}^{s}, \\
u^{\lambda^{\prime}} \rightarrow u \quad \text { weakly in } L_{T}^{p}\left(J^{-\delta} L^{q}\right)_{x}, \\
u^{\lambda^{\prime}} \rightarrow u \quad \text { in } L_{T}^{p} L_{\text {loc }, x}^{2},
\end{gathered}
$$

as $\lambda^{\prime} \rightarrow 0$. As for the convergence (3.8), we refer to [16, Ch. III, Theorem 2.1]. Using convergence above, we are able to observe that the limit $u$ satisfy (MSM) in the distribution sense. In fact, we observe that $u_{j}^{\lambda}$ satisfy 


$$
\begin{aligned}
\frac{d}{d t}\left\langle u_{j}^{\lambda}, \varphi\right\rangle+ & \left\langle u_{j}^{\lambda}, \Delta \varphi\right\rangle=-2 i\left\langle A\left[u^{\lambda}\right] u_{j}^{\lambda}, \nabla \varphi\right\rangle \\
& +\left\langle B\left[u^{\lambda}\right] u_{j}^{\lambda}, \varphi\right\rangle+\left\langle\left|A\left[u^{\lambda}\right]\right|^{2} u_{j}^{\lambda}, \varphi\right\rangle \pm i\left\langle\operatorname{Im}\left(u_{1}^{\lambda} \bar{u}_{2}^{\lambda}\right) u_{j^{\prime}}^{\lambda}, \varphi\right\rangle
\end{aligned}
$$

for $\varphi \in C_{0}^{\infty}\left(\mathbf{R}^{2}\right), j=1,2$, where $j^{\prime}=2$ if $j=1$, and $j^{\prime}=1$ if $j=2$. As for the convergence of the first term on the right hand side of (3.9), we consider

$$
\begin{aligned}
& \left\langle A\left[u^{\lambda}\right] u_{j}^{\lambda}, \nabla \varphi\right\rangle-\left\langle A[u] u_{j}, \nabla \varphi\right\rangle \\
& =\left\langle A\left[u^{\lambda}\right]\left(u_{j}^{\lambda}-u_{j}\right), \nabla \varphi\right\rangle+\left\langle\left(A\left[u^{\lambda}\right]-A[u]\right) u_{j}^{\lambda}, \nabla \varphi\right\rangle .
\end{aligned}
$$

Now we set $\operatorname{supp} \varphi \subset B_{R} \equiv\left\{x \in \mathbf{R}^{n} ;|x| \leq R\right\}$. Then, applying (2.5) we obtain

$$
\left|\left\langle A\left[u^{\lambda}\right]\left(u_{j}^{\lambda}-u_{j}\right), \nabla \varphi\right\rangle\right| \lesssim\left\|u^{\lambda}\right\|_{L^{2}}\left\|J^{\delta} u^{\lambda}\right\|_{L^{q}}\left\|u^{\lambda}-u\right\|_{L^{2}\left(B_{R}\right)}\|\nabla \varphi\|_{L^{2}}
$$

Similarly, we have

$$
\left|\left\langle\left(A\left[u^{\lambda}\right]-A[u]\right) u_{j}^{\lambda}, \nabla \varphi\right\rangle\right| \lesssim\left\|A\left[u^{\lambda}\right]-A[u]\right\|_{L^{q}\left(B_{R}\right)}\|u\|_{L^{2}}\|\nabla \varphi\|_{L^{p}},
$$

where $1 / q+1 / p=1 / 2$. To show the convergence, we set $L \geq 2 R$ to estimate

$$
\begin{aligned}
& \left\|A\left[u^{\lambda}\right]-A[u]\right\|_{L^{q}\left(B_{R}\right)} \\
& \lesssim\left\|I_{1}\left\{\left(\left|u^{\lambda}\right|+|u|\right)\left|u^{\lambda}-u\right| \chi_{B_{L}}\right\}\right\|_{L^{q}}+\left\|I_{1}\left\{\left(\left|u^{\lambda}\right|^{2}+|u|^{2}\right) \chi_{B_{L}^{c}}\right\}\right\|_{L^{q}\left(B_{R}\right)} \\
& \lesssim\left\|\left(\left|u^{\lambda}\right|+|u|\right)\left|u^{\lambda}-u\right| \chi_{B_{L}}\right\|_{L^{r}}+R^{n / q}\left\|I_{1}\left\{\left(\left|u^{\lambda}\right|^{2}+|u|^{2}\right) \chi_{B_{L}^{c}}\right\}\right\|_{L^{\infty}\left(B_{R}\right)} \\
& \lesssim\left(\left\|u^{\lambda}\right\|_{L^{q}}+\|u\|_{L^{q}}\right)\left\|u^{\lambda}-u\right\|_{L^{2}\left(B_{L}\right)}+R^{n / q}\left(\left\|u^{\lambda}\right\|_{L^{2}}^{2}+\|u\|_{L^{2}}^{2}\right) / L,
\end{aligned}
$$

where $B_{L}^{c} \equiv\left\{x \in \mathbf{R}^{n} ;|x|>L\right\}, r$ is determined by $1 / q=1 / r-1 / 2$, and $\chi_{E}$ denotes the characteristic function of a set $E$. For the last inequality above, we used the estimate

$$
\begin{aligned}
I_{1}\left\{\left(\left|u^{\lambda}\right|^{2}+|u|^{2}\right) \chi_{B_{L}^{c}}\right\}(x) & =C \int_{|y|>L} \frac{1}{|x-y|}\left(\left|u^{\lambda}(y)\right|^{2}+|u(y)|^{2}\right) d y \\
& \leq \frac{C}{L} \int\left(\left|u^{\lambda}(y)\right|^{2}+|u(y)|^{2}\right) d y,
\end{aligned}
$$

for $|x| \leq R \leq L / 2$, since $|x-y| \geq|y|-|x| \geq L / 2$. Thus, using the uniform bound (3.5), (3.6), we obtain

$$
\begin{aligned}
& \left|\int_{0}^{T}\left\langle A\left[u^{\lambda^{\prime}}\right] u_{j}^{\lambda^{\prime}}, \nabla \varphi\right\rangle \psi(t) d t-\int_{0}^{T}\left\langle A[u] u_{j}, \nabla \varphi\right\rangle \psi(t) d t\right| \\
& \lesssim\left\|u^{\lambda^{\prime}}-u\right\|_{L_{T}^{2} L^{2}\left(B_{L}\right)}+1 / L,
\end{aligned}
$$

which converges to zero as $\lambda^{\prime} \rightarrow 0, L \rightarrow \infty$. The convergence of other terms of (3.9) is also proved in the same manner.

Remark 3.2. It is worth noticing that the solution $u$ also belongs to $C\left([0, T] ; H^{s^{\prime}}\right)$ for $s^{\prime}<s$, since $\partial_{t} u \in L_{T}^{p / 2} H_{x}^{-2}$. 


\section{Uniqueness of $H^{1}$ solution}

In this section, we give the proof of Theorem 1.3. For the proof, the following Gagliardo-Nirenberg inequality with the specific constant, which we refer to $[14$, Lemma 2], is essentially used.

Lemma 4.1. For $2 \leq q<\infty$, we have

$$
\|u\|_{L^{q}\left(\mathbf{R}^{2}\right)} \leq(4 \pi)^{(2-q) / 2 q}(q / 2)^{1 / 2}\|u\|_{L^{2}\left(\mathbf{R}^{2}\right)}^{2 / q}\|\nabla u\|_{L^{2}\left(\mathbf{R}^{2}\right)}^{1-2 / q} .
$$

Proof of Theorem 1.3. If we set $w_{j}=u_{j}-v_{j}$, then $w_{j}$ satisfies

$$
\begin{aligned}
\left(i \partial_{t}+\Delta\right) w_{j}=- & 2 i A[u] \cdot \nabla w_{j}+B[u] w_{j}+|A[u]|^{2} w_{j} \pm i \operatorname{Im}\left(u_{1} \bar{u}_{2}\right) w_{j^{\prime}} \\
& -2 i(A[u]-A[v]) \cdot \nabla v_{j}+(B[u]-B[v]) v_{j} \\
& +\left(|A[u]|^{2}-|A[v]|^{2}\right) v_{j} \pm i\left(\operatorname{Im}\left(u_{1} \bar{u}_{2}\right)-\operatorname{Im}\left(v_{1} \bar{v}_{2}\right)\right) v_{j^{\prime}}
\end{aligned}
$$

for $j=1,2$, where $j^{\prime}$ denotes 2 if $j=1,1$ if $j=2$. Multiplying $\overline{w_{j}}$ by the equation (4.1), integrating over $\mathbf{R}^{n}$, and then taking the imaginary part, we obtain

$$
\partial_{t}\|w(t)\|_{L^{2}}^{2} \lesssim q M^{2+4 / q}\left(1+M^{2}\right)\|w(t)\|_{L^{2}}^{2(1-2 / q)}
$$

for $q>2$. Note that as we shall see below we finally take the limit $q \rightarrow \infty$, so it is important to investigate the dependence on $q$ of the constant appeared in the estimate. Once we obtain (4.2), then substituting $\varepsilon=2 / q$ we have

$$
\frac{1}{\varepsilon} \partial_{t}\|w(t)\|_{L^{2}}^{2 \varepsilon} \lesssim \frac{1}{\varepsilon} M^{2+2 \varepsilon}\left(1+M^{2}\right)
$$

Since $\|w(0)\|_{L^{2}}=0$, we obtain

$$
\|w(t)\|_{L^{2}} \leq\left(C M^{2+2 \varepsilon}\left(1+M^{2}\right) T\right)^{1 / 2 \varepsilon}
$$

for $0 \leq t \leq T$. Thus, if we take $T^{\prime}$ satisfying $C M^{2+2 \varepsilon}\left(1+M^{2}\right) T^{\prime}<1$, then letting $\varepsilon \rightarrow 0$ we conclude that $\|w(t)\|_{L^{2}}=0$ for $0 \leq t \leq T^{\prime}$. Using this argument repeatedly, the uniqueness holds for $0 \leq t \leq T$.

In what follows, we devote to derive (4.2) by estimating the right hand side of (4.1). As for the first term, integrating by parts it is easy to see that

$$
\operatorname{Im}\left\{i \int A[u] \cdot\left(\nabla w_{j}\right) \overline{w_{j}} d x\right\}=\frac{1}{2} \int(\operatorname{div} A[u])\left|w_{j}\right|^{2} d x=0,
$$

since $\operatorname{div} A[u]=0$. The second and the third terms are also 0 , since $B[u]$ and $|A[u]|^{2}$ are real valued. To estimate other terms, we set $p, q$ satisfying

$$
1 / 2=1 / p+1 / q, \quad 2<p \leq 4, \quad 4 \leq q<\infty .
$$

Then, the fourth term is estimated by applying Lemma 4.1 as follows.

$$
\begin{aligned}
\left|\int \operatorname{Im}\left(u_{1} \bar{u}_{2}\right) w_{j^{\prime}} \bar{w}_{j} d x\right| & \leq\|u\|_{L^{q}}^{2}\|w\|_{L^{p}}^{2} \\
& \lesssim q\|u\|_{L^{2}}^{4 / q}\|\nabla u\|_{L^{2}}^{2-4 / q}\|w\|_{L^{2}}^{4 / p}\|\nabla w\|_{L^{2}}^{2-4 / p} \\
& \lesssim q M^{2+4 / q}\|w\|_{L^{2}}^{2-4 / q}
\end{aligned}
$$


since $4 / p=2-4 / q$, where $M$ is the upper bound of $H^{1}$ norm in the assumption.

As for the fifth term, we have

$$
\left|\int(A[u]-A[v]) \nabla v_{j} \bar{w}_{j} d x\right| \lesssim\|A[u]-A[v]\|_{L^{q}}\|\nabla v\|_{L^{2}}\|w\|_{L^{p}} .
$$

Since $\left|\operatorname{Im}\left(u_{2} \bar{u}_{1}\right)-\operatorname{Im}\left(v_{2} \bar{v}_{1}\right)\right| \leq(|u|+|v|)|w|$, we have

$$
|A[u]-A[v]| \lesssim I_{1}((|u|+|v|)|w|) .
$$

Then, applying Lemma 2.3 we obtain

$$
\begin{aligned}
\|A[u]-A[v]\|_{L^{q}} & \lesssim\|(|u|+|v|)|w|\|_{L^{r}} \\
& \lesssim\left(\|u\|_{L^{q}}+\|v\|_{L^{q}}\right)\|w\|_{L^{2}}
\end{aligned}
$$

where $r$ is determined by $1 / q=1 / r-1 / 2$. Thus, applying Lemma 4.1 again, the right hand side of (4.3) is estimated by a constant multiple of

$$
q^{1 / 2} M^{2+2 / q}\|w\|_{L^{2}}^{2-2 / q} \leq q^{1 / 2} M^{2+4 / q}\|w\|_{L^{2}}^{2-4 / q} .
$$

Similarly, since $\left|\operatorname{Re}\left(u_{j} \bar{u}_{k}\right)-\operatorname{Re}\left(v_{j} \bar{v}_{k}\right)\right| \leq(|u|+|v|)|w|$, we have

$$
\|B[u]-B[v]\|_{L^{2}} \lesssim\left(\|u\|_{L^{q}}+\|v\|_{L^{q}}\right)\|w\|_{L^{p}} .
$$

Thus, we obtain

$$
\begin{aligned}
\left|\int(B[u]-B[v]) v_{j} \bar{w}_{j} d x\right| & \leq\|B[u]-B[v]\|_{L^{2}}\|v\|_{L^{q}}\|w\|_{L^{p}} \\
& \lesssim\left(\|u\|_{L^{q}}+\|v\|_{L^{q}}\right)\|v\|_{L^{q}}\|w\|_{L^{p}}^{2} .
\end{aligned}
$$

Then, we are able to estimate in the same way as the fourth term. We note that the eighth term is estimated in the same manner.

Finally, as for the seventh term, we use (4.4) to estimate

$$
\begin{aligned}
& \left|\int\left(|A[u]|^{2}-|A[v]|^{2}\right) v_{j} \bar{w}_{j} d x\right| \\
& \lesssim\left(\|A[u]\|_{L^{q}}+\|A[v]\|_{L^{q}}\right)\|A[u]-A[v]\|_{L^{q}}\|v\|_{L^{p}}\|w\|_{L^{p}} \\
& \lesssim M\left(\|u\|_{L^{q}}^{2}+\|v\|_{L^{q}}^{2}\right)\|v\|_{L^{p}}\|w\|_{L^{p}}\|w\|_{L^{2}} \\
& \lesssim q M^{4+2 / q}\|w\|_{L^{2}}^{2-2 / q} \\
& \lesssim q M^{4+4 / q}\|w\|_{L^{2}}^{2-4 / q}
\end{aligned}
$$

Therefore, we obtain (4.2).

\section{References}

[1] L. Bergé, A. de Bouard, J. C. Saut, Blowing up time-dependent solutions of the planar, Chern-Simons gauged nonlinear Schrödinger equation, Nonlinearity 8 (1995), 235-253.

[2] T. Cazenave, Semilinear Schrödinger equations, Courant Lecture Notes in Mathematics 10, American Mathematical Society (2003).

[3] D. Chae, On the well-posedness of the Euler equations in the Triebel-Lizorkin spaces, Comm. Pure Appl. Math. 55 (2002), 654-678.

[4] N.-H. Chang, J. Shatah, K. Uhlenbeck, Schödinger maps, Comm. Pure Appl. Math. 53 (2000), 590-602. 
[5] W. Ding, Y. Wang, Local Schrödinger flow into Kähler manifolds, Sci. China Ser. A 44 (2001), 1446-1464.

[6] M. G. Grillakis, V. Stefanopoulos, Lagrangian formulation, energy estimates, and the Schrödinger map problem, Comm. Partial Differential Equations 27 (2002), 1845-1877.

[7] C. E. Kenig, private communication.

[8] C. E. Kenig, K. D. Koenig, On the local well-posedness of the Benjamin-Ono and modified Benjamin-Ono equations, Math. Res. Lett. 10 (2003), 879-895.

[9] C. E. Kenig, G. Ponce, L. Vega, On the initial value problem for the Ishimori system, Ann. Henri Poincaré 1 (2000), 341-384.

[10] H. Koch, N. Tzvetkov, On the local well-posedness of the Benjamin-Ono equation in $H^{s}(\mathbb{R})$, Int. Math. Res. Not. 26 (2003), 1449-1464.

[11] A. Nahmod, A. Stefanov, K. Uhlenbeck, On Schrödinger maps, Comm. Pure Appl. Math. 56 (2003), 114-151.

[12] A. Nahmod, A. Stefanov, K. Uhlenbeck, Erratum: On Schrödinger maps [Comm. Pure Appl. Math. 56 (2003), 114-151], Comm. Pure Appl. Math. 57 (2004), 833-839.

[13] M. Nakamura, T. Wada, Local well-posedness for the Maxwell-Schrödinger equation, to appear in Mathematische Annalen.

[14] T. Ogawa, A proof of Trudinger's inequality and its application to nonlinear Schrödinger equations, Nonlinear Anal. 14 (1990), 765-769.

[15] E. M. Stein, Singular integrals and differentiability properties of functions, Princeton Mathematical Series 30, Princeton University Press (1970).

[16] R. Temam, Navier-Stokes equations, Theory and numerical analysis, Revised edition, Studies in Mathematics and its Applications 2, North-Holland Publishing Co. (1979).

[17] M. V. Vladimirov, On the solvability of a mixed problem for a nonlinear equation of Schrödinger type, Soviet Math. Dokl. 29 (1984), 281-284.

Department of Mathematics, Kyoto University, Kyoto 606-8502, Japan

E-mail address: jkato@math.kyoto-u.ac.jp 